\title{
Generating nonclassical quantum input field states with modulating filters
}

\author{
John E Gough ${ }^{1 *}$ and Guofeng Zhang ${ }^{2}$
}

\author{
“Correspondence: jug@aber.ac.uk \\ 'Department of Physics, \\ Aberystwyth University, \\ Aberystwyth, Wales SY23 2BZ, UK \\ Full list of author information is \\ available at the end of the article
}

\begin{abstract}
We give explicit constructions of quantum dynamical filters which generate nonclassical states (coherent states, cat states, shaped single and multi-photon states) of quantum optical fields as inputs to general quantum Markov systems. The filters will be quantum harmonic oscillators damped by the input fields, and we exploit the fact that the cascaded filter and system will have a Lindbladian that is naturally Wick-ordered in the filter modes. In particular the initialization of the modulating filter will determine the signal state generated.
\end{abstract}

Keywords: quantum modulating filter; nonclassical states; measurement filtering

\section{Introduction}

There has been considerable progress in the generation of nonclassical states of light such as shaped single photons [1-6], cat states [7-9], etc., and this has been proposed for several quantum technologies [10-18]. Here we propose the use of quantum mechanical modulating filters prepared in nonclassical states which serve to generate nonclassical quantum noise output from vacuum input, and that may then be used to drive an open quantum system. The effect, after tracing out the modulator, is equivalent to driving the system by an input field in a nonclassical state, see Figure 1.

Historical, the concept of coloring white noises has enjoyed much application in control engineering, and in particular signal processing. A white noise input, say corresponding to the derivative of a Wiener process $B(t)$, may be converted into a colored process $Y(t)=\int h(t-s) d B(s)$ where $h$ is a causal kernel function. In practice this convolution may be physically implementable by passing the input through a dynamical system, such as an electronic circuit, an obtaining $Y$ as output. The resulting output will have a nonflat spectrum $S_{Y}(\omega) \equiv|H(\omega)|^{2}$, where $H(\omega)$ is the Fourier transform of the kernel $h$, see for instance [13]. However, the concept is still useful as a theoretical construct in modelling systems driven by colored noise, as it allows an extended model with a white noise input.

The idea has been extended to quantum systems, and at its simplest corresponds to cascading an ancillary system (the filter) in front of the system, a concept going back to Carmichael, [14]. A systematic study of quantum coloring filters was initiated in [15] by one of the authors. More recently, finite-level ancillas were proposed to generate multiphoton states for quantum input processes [16]. In this setting, the dynamical and filtering equations took on a matrix form determined by the ancilla space. However, due to the

(c) 2015 Gough and Zhang. This article is distributed under the terms of the Creative Commons Attribution 4.0 International License (http://creativecommons.org/licenses/by/4.0/), which permits unrestricted use, distribution, and reproduction in any medium, provided you give appropriate credit to the original author(s) and the source, provide a link to the Creative Commons license, and indicate if changes were made. 
(A) Nonclassical input state

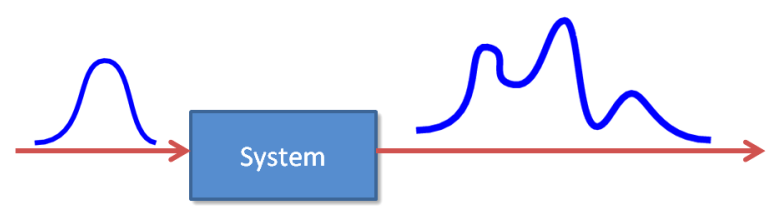

(B) Vacuum input state

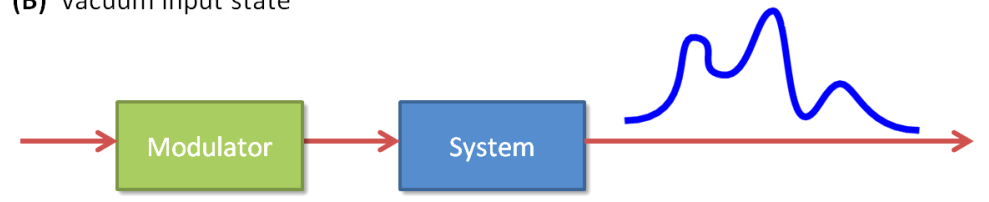

Figure 1 System with nonclassical input; a coloring filter (modulator) is used to convert vacuum noise input into a desired nonclassical input. Tracing out the modulating filter leads to the same master equation.

choice of couplings (raising and lowering of the ancilla levels) the class of multi-photon states obtained had a chronological ordering property of the photon one-particle states which was not the intended form of the multi-photon state. Or alternative here is to use linear quantum dynamical models as ancilla. We should also mention recent work of Xue et al. [17] who have treated the Belavkin filtering problem for Ornstein-Uhlenbeck noise input: this input may be readily modelled as output of a linear system such as a cavity mode driven by white noise quantum input processes.

Notations Denote by $\mathcal{F}_{n}$ the span of all symmetrized vectors of the form $f_{1} \hat{\otimes} \cdots \hat{\otimes} f_{n}=$ $\frac{1}{n !} \sum_{\sigma} f_{\sigma(1)} \otimes \cdots \otimes f_{\sigma(n)}$ where $f_{1}, \ldots, f_{n}$ lie in a one-particle Hilbert space $\mathcal{V}$, and the sum is over all permutations $\sigma$ of the $n$ indices. The Boson Fock space over $\mathcal{V}$ is then the direct sum $\mathcal{F}=\bigoplus_{n=0}^{\infty} \mathcal{F}_{n}$, with $\mathcal{F}_{0}$ spanned by the vacuum vector $\mid$ vac $\rangle$.

The creation, annihilation and conservation operators are then given by $\left(\widehat{f}_{j}\right.$ indicating the omission of term $f_{j}$ )

$$
\begin{aligned}
& B(g)^{*} f_{1} \hat{\otimes} \cdots \hat{\otimes} f_{n}=\sqrt{n+1} g \hat{\otimes} f_{1} \hat{\otimes} \cdots \hat{\otimes} f_{n}, \\
& B(g) f_{1} \hat{\otimes} \cdots \hat{\otimes} f_{n}=\frac{1}{\sqrt{n}} \sum_{j=1}^{n}\left\langle g \mid f_{j}\right\rangle f_{1} \hat{\otimes} \cdots \hat{\otimes} \widehat{f_{j}} \hat{\otimes} \cdots \hat{\otimes} f_{n}, \\
& \Lambda(T) f_{1} \hat{\otimes} \cdots \hat{\otimes} f_{n}=\sum_{j=1}^{n} f_{1} \hat{\otimes} \cdots \hat{\otimes}\left(T f_{j}\right) \hat{\otimes} \cdots \hat{\otimes} f_{n},
\end{aligned}
$$

and they map $\mathcal{F}_{n}$ to $\mathcal{F}_{n+1}, \mathcal{F}_{n-1}$ and $\mathcal{F}_{n}$ respectively.

Given a complete orthonormal basis $\left\{e_{1}, e_{2}, \ldots\right\}$ for $\mathcal{V}$, we obtain a complete orthonormal basis for $\mathcal{F}$ by setting

$$
|\mathbf{n}\rangle=\sqrt{\frac{n !}{n_{1} ! n_{2} ! \cdots}} \widehat{\bigotimes}_{k} e_{k}^{\otimes n_{k}} \equiv \prod_{k=1}^{\infty} \frac{1}{\sqrt{n_{k} !}} B\left(e_{k}\right)^{* n_{k}}|\mathrm{vac}\rangle,
$$


where $\mathbf{n}=\left(n_{1}, n_{2}, \ldots\right)$ is a sequence of occupation numbers and $n=\sum_{k} n_{k}$. The state $|\mathbf{n}\rangle$ corresponds to having $n_{k}$ photons in the state $e_{k}$ for each $k$.

For $\mathcal{V}=L^{2}[0, \infty)$, the space of square-integrable functions $\xi(t)$ in $t \geq 0$, we introduce the annihilation process $B_{t}=B\left(\chi_{[0, t]}\right)$ where $\chi_{[0, t]}$ is the function equal to unity on the interval 0 to $t$, and zero otherwise. The Itō differential $d B_{t}$ has the action $d B_{t}|\mathbf{n}\rangle=$ $\left.\sum_{k=1}^{\infty} \sqrt{n_{k}} \mid n_{1}, \ldots, n_{k}-1, \ldots\right) e_{k}(t) d t$, where, for convenience, we may assume an orthonormal basis of continuous test functions $e_{k}$. For nonorthonormal states we have

$$
d B_{t} f_{1} \hat{\otimes} \cdots \hat{\otimes} f_{n}=\frac{1}{\sqrt{n}} \sum_{j} f_{1} \hat{\otimes} \cdots \hat{\otimes} \widehat{f}_{j} \hat{\otimes} \cdots \hat{\otimes} f_{n} f_{j}(t) d t .
$$

For convenience we consider a single quantum input process.

Now fix a quantum mechanical system with Hilbert space $\mathfrak{h}_{0}$, called the initial space, then an open system is described by the triple of operators $G \sim(S, L, H)$ on $\mathfrak{h}_{0}$ - with $S$ the unitary scattering matrix, $L$ the collapse, or coupling, operator and $H$ the Hamiltonian - which fixes the open dynamical unitary evolution $U(t)$ on $\mathfrak{h}_{0} \otimes \mathfrak{F}$ as the solution to the quantum stochastic differential equation [19]

$$
d U_{t}=\left\{(S-I) \otimes d \Lambda_{t}+L \otimes d B_{t}^{*}-L^{*} S \otimes d B_{t}-\left(\frac{1}{2} L^{*} L+i H\right) \otimes d t\right\} U_{t}
$$

and in the Heisenberg picture we set $j_{t}(X)=U_{t}^{*} X \otimes I U_{t}$ so that

$$
\begin{aligned}
d j_{t}(X)= & j_{t}\left(\mathcal{L}_{11} X\right) \otimes d \Lambda_{t}+j_{t}\left(\mathcal{L}_{10} X\right) \otimes d B_{t}^{*} \\
& +j_{t}\left(\mathcal{L}_{01} X\right) \otimes d B_{t}+j_{t}\left(\mathcal{L}_{00} X\right) \otimes d t
\end{aligned}
$$

where we have the Evans-Hudson super-operators [20]

$$
\begin{aligned}
& \mathcal{L}_{00} X=\frac{1}{2} L^{*}[X, L]+\frac{1}{2}\left[L^{*}, X\right] L-i[X, H], \\
& \mathcal{L}_{10} X=S^{*}[X, H], \quad \mathcal{L}_{01} X=\left[L^{*}, X\right] S, \\
& \mathcal{L}_{11} X=S^{*} X S-X .
\end{aligned}
$$

The output processes are given by the formula

$$
B_{t}^{\text {out }}=U^{*}(t) B(t) U(t)
$$

and we have $d B_{t}^{\text {out }}=j_{t}(S) d B_{t}+j_{t}(L) d t$.

Finally we recall that there is the natural factorization $\mathfrak{F}=\mathfrak{F}_{t}^{-} \otimes \mathfrak{F}_{t}^{+}$of the Fock space into past and future Fock spaces for each $t>0$ [19].

Definition 1 (from [19]) An operator on a tensor product $\mathfrak{h}_{1} \otimes \mathfrak{h}_{2}$ is the ampliation of an operator $X_{1}$ on $\mathfrak{h}_{1}$ if it takes the form $X_{1} \otimes I_{2}$. A quantum stochastic process $(X(t))_{t \geq 0}$ is adapted if, for each $t>0$, it is the ampliation of an operator on the past space $\mathfrak{h}_{0} \otimes \mathfrak{F}_{t}^{-}$to the full space $\mathfrak{h}_{0} \otimes \mathfrak{F}$.

The unitary evolution process $(U(t))_{t \geq 0}$ is adapted, as will be the Heisenberg dynamical process $\left(j_{t}(X)\right)_{t \geq 0}$, for each initial choice of operator $X$. The following formula will be used extensively. 
Lemma Let $(X(t))_{t \geq 0}$ be a quantum stochastic integral process of the form

$$
X(t)=X_{0} \otimes I_{\mathfrak{F}}+\int_{0}^{t}\left[x_{00}(s) d s+x_{10}(s) d B_{s}^{*}+x_{01}(s) d B_{s}+x_{11}(s) d \Lambda_{s}\right],
$$

where the $\left(x_{\alpha \beta}(t)\right)_{t \geq 0}$ are adapted processes. Then

$$
\begin{aligned}
d\left[U^{*}(t) X(t) U(t)\right]= & U^{*}(t)\left\{\left[\mathcal{L}_{00}\left(X_{0}\right)+x_{00}(t)+L^{*} x_{10}+x_{01} L+x_{11} L\right] d t\right. \\
& +\left[\mathcal{L}_{10}\left(X_{0}\right)+S^{*} x_{10}(t)+S^{*} x_{11} L\right] d B_{t}^{*} \\
& +\left[\mathcal{L}_{01}\left(X_{0}\right)+x_{01}(t) S+L^{*} x_{11} S\right] d B_{t} \\
& \left.+\left[\mathcal{L}_{11}\left(X_{0}\right)+S^{*} x_{11}(t) S\right] d \Lambda_{t}\right\} U(t) .
\end{aligned}
$$

The proof is a routine application of the quantum stochastic calculus [19]. We note that if we set the $\left(x_{\alpha \beta}(t)\right)_{t \geq 0}$ equal to zero then we recover the standard Lindblad-Heisenberg equations of motion for $U^{*}(t)\left(X_{0} \otimes I_{\mathfrak{F}}\right) U(t)$ : that is the noisy dynamics of the system observable with initial value $X_{0}$. Conversely setting $X_{0}=0$ and taking the $\left(x_{\alpha \beta}(t)\right)_{t \geq 0}$ to be constants leads to the input-output relation. Equation (5) therefore contains general information about evolution of both system observables and field observables.

\section{Modulating filter}

Our strategy is to employ a modulating filter $M$ to process vacuum input and to feed this forward to the system. In principle, the modulator and system are run in series as a single Markov component driven by vacuum input, as in Figure 1. Tracing out the modulator degrees of freedom leads to an effective model which leads to the same statistical model as a nonvacuum input to the system. We shall show below how to realize different nonclassical driving fields in this way. In our proposal we consider a linear passive system as modulator: physically corresponding to modes in a cavity. The choice of (time-dependent) coupling operators describing the modulator will be important in shaping the output, however, in this set-up the crucial element determining nonvacuum statistics will be the initial state $\phi_{0} \in \mathfrak{h}_{M}$ of the modulator.

We consider our system $G \sim(S, L, H)$ which is driven by the output of a modulator $M \sim$ $\left(I, L_{M}, H_{M}\right)$ which itself is driven by vacuum noise. The modulator and system in series is described by the series product $[21,22]$ on the joint space $\mathfrak{h}_{M} \otimes \mathfrak{h}_{G}$

$$
\widetilde{G}=G \triangleleft M \sim\left(I \otimes S, I \otimes L+L_{M} \otimes S, I \otimes H+H_{M} \otimes I+\operatorname{Im}\left\{L_{M} \otimes L^{*} S\right\}\right) .
$$

Let us denote by $\widetilde{U}_{t}$ the joint unitary generated by $\widetilde{G}$. This is a unitary adapted process with initial space $\mathfrak{h}_{0}=\mathfrak{h}_{M} \otimes \mathfrak{h}_{G}$.

Definition 2 Let $G$ determine an open quantum system and let $\Xi \in \mathfrak{F}$ be a state of the input field. A modulator $M$ with initial state $\phi_{0}$ and vacuum input is said to replicate the open system if we have (with $\left|\Psi_{0}\right\rangle=\mid \phi_{0} \otimes \psi_{0} \otimes$ vac $\rangle$ )

$$
\left\langle\Psi_{0}\left|\widetilde{U}(t)^{*}\left(I_{M} \otimes X(t)\right) \widetilde{U}(t)\right| \Psi_{0}\right\rangle=\left\langle\psi_{0} \otimes \Xi\left|U(t)^{*} X(t) U(t)\right| \psi_{0} \otimes \Xi\right\rangle
$$

for every adapted process $(X(t))_{t \geq 0}$ on $\mathfrak{h}_{G} \otimes \mathfrak{F}$ and all $\psi_{0} \in \mathfrak{h}$. 


\subsection{The cascaded Lindbladian}

The total Lindbladian corresponding to $\widetilde{G}$ is

$$
\widetilde{\mathcal{L}}(A \otimes X)=\mathcal{L}_{M}(A) \otimes X+\sum_{\mu=0,1} \sum_{\nu=0,1}\left[L_{M}^{*}\right]^{\mu} A\left[L_{M}\right]^{\nu} \otimes \mathcal{L}_{\mu \nu} X
$$

where $\mathcal{L}_{M} A=\frac{1}{2}\left[L_{M}^{*}, A\right] L_{M}+\frac{1}{2} L_{M}^{*}\left[A, L_{M}\right]-i\left[A, H_{M}\right]$ is the modulator Lindbladian.

\subsection{Oscillator mode modulators}

Our interest will be in modulators that are linear passive systems. To this end, we begin with the simplest model of a single Boson mode $a$ (say a cavity mode) as modulator, and set

$$
H_{M}=\omega(t) a^{*} a, L_{M}=\lambda(t) a .
$$

For simplicity we shall take $\omega(t) \equiv 0$ and $\lambda$ to be a complex-valued time-dependent damping parameter.

A key feature of equation (8) when $L_{M}=\lambda(t) a$ is that the $a$ and $a^{*}$ appear in Wick ordered form about $A$. We now exploit this property.

To compute expectations, we introduce the operator

$$
\tilde{a}_{t} \triangleq \widetilde{U}_{t}^{*}(a \otimes I) \widetilde{U}_{t}
$$

and observe that $d \widetilde{a}_{t}=-z(t) \widetilde{a}_{t} d t-\lambda(t)^{*} d B_{t}$ where we have the complex damping $z(t)=$ $\frac{1}{2}|\lambda(t)|^{2}+i \omega(t)$. The solution to this is the operator

$$
\tilde{a}_{t}=e^{-\zeta(t)} a-\int_{0}^{t} \lambda(s)^{*} e^{\zeta(s)-\zeta(t)} d B_{s}
$$

with $\zeta(t)=\int_{0}^{t} z(s) d s$.

Setting $\left|\Psi_{0}\right\rangle=\mid \phi_{0} \otimes \psi_{0} \otimes$ vac $\rangle$, we have

$$
\begin{aligned}
\frac{d}{d t} & \left\langle\Psi_{0}\left|\widetilde{U}(t)^{*}\left(I_{M} \otimes X \otimes I_{\mathfrak{F}}\right) \widetilde{U}(t)\right| \Psi_{0}\right\rangle \\
& =\sum_{\mu, \nu=0,1}\left\langle\Psi_{0}\left|\widetilde{U}(t)^{*}\left(\left[\lambda(t)^{*} a^{*}\right]^{\mu}[\lambda(t) a]^{\nu} \otimes \mathcal{L}_{\mu \nu} X\right) \widetilde{U}(t)\right| \Psi_{0}\right\rangle \\
& =\sum_{\mu, \nu=0,1}\left\langle\Psi_{0}\left|\left[\lambda(t)^{*} \widetilde{a}_{t}^{*}\right]^{\mu} \widetilde{U}(t)^{*}\left(I_{M} \otimes \mathcal{L}_{\mu \nu} X\right) \widetilde{U}(t)\left[\lambda(t) \widetilde{a}_{t}\right]^{\nu}\right| \Psi_{0}\right\rangle \\
& =\sum_{\mu, \nu=0,1}\left[\xi(t)^{*}\right]^{\mu}[\xi(t)]^{\nu}\left\langle[a]^{\mu} \Psi_{0}\left|\widetilde{U}_{t}^{*}\left(I \otimes \mathcal{L}_{\mu \nu} X \otimes I\right) \widetilde{U}_{t}\right|[a]^{\nu} \Psi_{0}\right\rangle,
\end{aligned}
$$

where

$$
\xi(t)=\lambda(t) e^{-\zeta(t)} .
$$




\subsection{Generating shaped 1-photon fields}

The problem we ideally wish to solve is how to generate a desired pulse shape $\xi$, and this means choosing the correct $\lambda$. It will be required that $\xi$ be normalized, that is, $\int_{0}^{\infty}|\xi(t)|^{2} d t=1$. Now let us set $\mathrm{w}(t)=\exp \left\{-\int_{0}^{t}|\lambda(s)|^{2} d s\right\}$, then

$$
\frac{d}{d t} \mathrm{w}(t)=-|\lambda(t)|^{2} \exp \left\{-\int_{0}^{t}|\lambda(s)|^{2} d s\right\} \equiv-|\xi(t)|^{2}
$$

and, imposing the correct initial condition $w(0)=1$, we obtain

$$
\mathrm{w}(t) \equiv \int_{t}^{\infty}|\xi(s)|^{2} d s
$$

Again taking $\omega(t) \equiv 0$ for simplicity, we find $z(t) \equiv \frac{1}{2}|\lambda(t)|^{2}$, real-valued. As we are given $\xi$ normalized, we see that the appropriate choice for $\lambda$ is

$$
\lambda(t)=\frac{1}{\sqrt{\mathrm{w}(t)}} \xi(t)
$$

with $w$ given by (12). An additional phase term will appear if we have $\omega(t)$ nonzero.

\subsection{Replicating nonvacuum input}

Let $G \sim(S, L, H)$ and $M \sim\left(I_{M}, \lambda a, \omega a^{*} a\right)$ and let $(X(t))_{t \geq 0}$ be a quantum stochastic integral process on $\mathfrak{h}_{G} \otimes \mathfrak{F}$, as in (4) then we may generalize (10) to get

$$
\begin{aligned}
\frac{d}{d t}\left\langle\Psi_{0}\left|\widetilde{U}(t)^{*}\left(I_{M} \otimes X(t)\right) \widetilde{U}(t)\right| \Psi_{0}\right\rangle \\
=\left\langle\Psi_{0}\left|\widetilde{U}(t)^{*} I_{M} \otimes\left(\mathcal{L}_{00}\left(X_{0}\right)+x_{00}+L^{*} x_{10}+x_{01} L+L^{*} x_{11} L\right) \widetilde{U}(t)\right| \Psi_{0}\right\rangle \\
\quad+\xi^{*}\left\langle\Psi_{0}\left|a^{*} \widetilde{U}(t)^{*} I_{M} \otimes\left(\mathcal{L}_{10}\left(X_{0}\right)+S^{*} x_{10}+S^{*} x_{11} L\right) \widetilde{U}(t)\right| \Psi_{0}\right\rangle \\
\quad+\xi\left\langle\Psi_{0}\left|\widetilde{U}(t)^{*} I_{M} \otimes\left(\mathcal{L}_{01}\left(X_{0}\right)+x_{01} S+L^{*} x_{11} S\right) \widetilde{U}(t) a\right| \Psi_{0}\right\rangle \\
\quad+\xi^{*} \xi\left\langle\Psi_{0}\left|a^{*} \widetilde{U}(t)^{*} I_{M} \otimes\left(\mathcal{L}_{11}\left(X_{0}\right)+S^{*} x_{11} S\right) \widetilde{U}(t) a\right| \Psi_{0}\right\rangle .
\end{aligned}
$$

This follows from (5) where we replace the $S$ and $L$ with the cascaded operators $I_{M} \otimes S$ and $I_{M} \otimes L+\lambda a \otimes S$.

For the modulator to replicate the dynamics with nonvacuum state $\Xi$, the derivative

$$
\frac{d}{d t}\left\langle\psi_{0} \otimes \Xi\left|U(t)^{*} X(t) U(t)\right| \psi_{0} \otimes \Xi\right\rangle
$$

must equal the corresponding expression (14) for any quantum stochastic integral process $X(t)$ on the Hilbert space $\mathfrak{h}_{G} \otimes \mathfrak{F}$. We may use (5) to show directly that this is computed from the following expectation

$$
\begin{aligned}
d\left\langle\psi_{0} \otimes \Xi\left|U(t)^{*} X(t) U(t)\right| \psi_{0} \otimes \Xi\right\rangle \\
=\left\langle\psi_{0} \otimes \Xi\right| U^{*}(t)\left\{\left[\mathcal{L}_{00}\left(X_{0}\right)+x_{00}(t)+L^{*} x_{10}+x_{01} L+x_{11} L\right] d t\right. \\
\quad+\left[\mathcal{L}_{10}\left(X_{0}\right)+S^{*} x_{10}(t)+S^{*} x_{11} L\right] d B_{t}^{*}
\end{aligned}
$$




$$
\begin{aligned}
& +\left[\mathcal{L}_{01}\left(X_{0}\right)+x_{01}(t) S+L^{*} x_{11} S\right] d B_{t} \\
& \left.+\left[\mathcal{L}_{11}\left(X_{0}\right)+S^{*} x_{11}(t) S\right] d \Lambda_{t}\right\} U(t)\left|\psi_{0} \otimes \Xi\right\rangle
\end{aligned}
$$

The modulator therefore replicates the nonvacuum input model.

\subsection{Replicating coherent states}

As a simple illustration let us show how we may construct a modulator that replicates a coherent state $|\beta\rangle$ for the input field, where $\beta(t)$ is a square integrable function of time $t \geq 0$. Note that

$$
d B_{t}|\beta\rangle=\beta(t)|\beta\rangle .
$$

We see that the equations (14) and (15) have structural similarities, and a first guess for the initial state of the modulator is another coherent state

$$
\phi_{0}=|\alpha\rangle,
$$

where $\alpha \in \mathbb{C}$ is the intensity of the mode coherent state. In this case $a \phi_{0}=\alpha \phi_{0}$ and (14) and (15) coincide for the choice

$$
\xi(t) \alpha \equiv \beta(t) .
$$

We therefore get the following result.

Theorem 1 The quantum open system $G \sim(S, L, H)$ driven by input in the continuousvariable coherent state $\Xi=|\beta\rangle$ is replicated by the single mode modulator of the linear form $M \sim\left(I_{M}, \lambda a, \omega a^{*} a\right)$ with the initial state $\phi_{0}=|\alpha\rangle$ for the modulator and with $\lambda(t)$ and $\omega(t)$ chosen so that (16) holds.

\section{Replicating multi-photon input}

\subsection{Fock state input fields}

The state of a single mode quantum input field corresponding to $n$ quanta with the same (normalized) one-particle test function $\xi \in L^{2}[0, \infty)$ is

$$
\xi^{\otimes n}=\frac{1}{\sqrt{n !}} B^{*}(\xi)^{n}|\mathrm{vac}\rangle .
$$

We see that the annihilator acts on such states as

$$
d B_{t} \xi^{\otimes n}=\sqrt{n} \xi(t) \xi^{\otimes n-1} d t .
$$

One of the consequences of this comes when we try and compute expectations of the form

$$
\left\langle\psi_{0} \otimes \xi^{\otimes n}\left|x_{01}(t) d B_{t}\right| \psi_{0} \otimes \xi^{\otimes n}\right\rangle
$$

which then equals

$$
\sqrt{n} \xi(t)\left\langle\psi_{0} \otimes \xi^{\otimes n}\left|x_{01}(t)\right| \psi_{0} \otimes \xi^{\otimes n-1}\right\rangle d t
$$


which is a matrix element between an $n$ photon state and an $n-1$ photon state. This feature will be typical, and so it is convenient to introduce general matrix elements

$$
M_{t}^{n_{l}, n_{r}}(X)=\left\langle\psi_{0} \otimes \xi^{\otimes n_{l}}\left|U(t)^{*} X(t) U(t)\right| \psi_{0} \otimes \xi^{\otimes n_{r}}\right\rangle,
$$

whenever $X(t)$ is a quantum stochastic integral and $n_{l}, n_{r} \geq 0$. We set $M_{t}^{n_{l}, n_{r}}(X) \equiv 0$ if we ever have $n_{l}$ or $n_{r}$ negative.

Taking $X$ to have the form (4), we see that (15) leads to

$$
\begin{aligned}
\frac{d}{d t} M_{t}^{n_{l}, n_{r}}(X)= & M_{t}^{n_{l}, n_{r}}\left(\mathcal{L}_{00}\left(X_{0}\right)+x_{00}(t)+L^{*} x_{10}+x_{01} L+x_{11} L\right) \\
& +\sqrt{n} \xi^{*}(t) M_{t}^{n_{l}-1, n_{r}}\left(\mathcal{L}_{10}\left(X_{0}\right)+S^{*} x_{10}(t)+S^{*} x_{11} L\right) \\
& +\sqrt{n} \xi(t) M_{t}^{n_{l}, n_{r}-1}\left(\mathcal{L}_{01}\left(X_{0}\right)+x_{01}(t) S+L^{*} x_{11} S\right) \\
& +n|\xi(t)|^{2} M_{t}^{n_{l}-1, n_{r}-1}\left(\mathcal{L}_{11}\left(X_{0}\right)+S^{*} x_{11}(t) S\right) .
\end{aligned}
$$

We note the hierarchical nature of these equations with the rate of change of $M_{t}^{n_{l}, n_{r}}(X)$ depending on lower order matrix elements.

Now let us introduce the single mode modulator, and let $|n\rangle$ be the number states for the oscillator mode $(n=0,1,2, \ldots)$. We may similarly introduce the matrix elements

$$
\widetilde{M}_{t}^{n_{l}, n_{r}}(X)=\left\langle n_{l} \otimes \psi_{0} \otimes \operatorname{vac}\left|\widetilde{U}(t)^{*}\left(I_{M} \otimes X(t)\right) U(t)\right| n_{r} \otimes \psi_{0} \otimes \mathrm{vac}\right\rangle,
$$

with $n_{l}, n_{r} \geq 0$, and $\widetilde{M}_{t}^{n_{l}, n_{r}}(X)=0$ if either index is negative.

From (14), we see that

$$
\begin{aligned}
\frac{d}{d t} \widetilde{M}_{t}^{n_{l}, n_{r}}(X)= & \widetilde{M}_{t}^{n_{l}, n_{r}}\left(\mathcal{L}_{00}\left(X_{0}\right)+x_{00}(t)+L^{*} x_{10}+x_{01} L+x_{11} L\right) \\
& +\sqrt{n} \xi^{*}(t) \widetilde{M}_{t}^{n_{l}-1, n_{r}}\left(\mathcal{L}_{10}\left(X_{0}\right)+S^{*} x_{10}(t)+S^{*} x_{11} L\right) \\
& +\sqrt{n} \xi(t) \widetilde{M}_{t}^{n_{l}, n_{r}-1}\left(\mathcal{L}_{01}\left(X_{0}\right)+x_{01}(t) S+L^{*} x_{11} S\right) \\
& +n|\xi(t)|^{2} \widetilde{M}_{t}^{n_{l}-1, n_{r}-1}\left(\mathcal{L}_{11}\left(X_{0}\right)+S^{*} x_{11}(t) S\right) .
\end{aligned}
$$

It follows that the systems of equations (17) and (18) are identical, and so we identify

$$
M_{t}^{n_{l}, n_{r}}(X) \equiv \widetilde{M}_{t}^{n_{l}, n_{r}}(X)
$$

for all quantum stochastic integral process $X(t)$ on the joint system and field. We summarize the result as follows.

Theorem 2 The quantum open system $G \sim(S, L, H)$ driven by input in the nonclassical state $\Xi=\xi^{\otimes n}$ is replicated by the single mode modulator of the linear form $M \sim$ $\left(I_{M}, \lambda(t) a, \omega(t) a^{*} a\right)$ with the initial state $\phi_{0}=|n\rangle$ for the modulator and with $\lambda(t)$ and $\omega(t)$ chosen so that (11) holds. 


\subsection{General multi-photon input fields}

To generate multi-photon input field state (assumed normalized)

$$
\Xi(\mathbf{n})=\widehat{\bigotimes}_{k=1}^{N} \xi_{k}^{\otimes n_{k}},
$$

where now the $\xi_{k}$ are distinct, we need a multimode cavity with several independent photon modes $a_{1}, \ldots, a_{N}$. The coupling operator may now be extended to

$$
L_{M}=\sum_{k} \lambda_{k}(t) a_{k}
$$

It is convenient to introduce the vectors

$$
\begin{aligned}
& \xi(t)=\left[\xi_{1}(t), \ldots, \xi_{N}(t)\right], \\
& \lambda(t)=\left[\lambda_{1}(t), \ldots, \lambda_{N}(t)\right], \\
& a=\left[\begin{array}{c}
a_{1} \\
\vdots \\
a_{N}
\end{array}\right]
\end{aligned}
$$

so that $L_{M} \equiv \lambda(t) a$. We consider the vector of time-evolved modes $\widetilde{a}_{t}=\tilde{U}_{t}^{*} a \widetilde{U}_{t}$ and from the Itō rules, we find

$$
d \tilde{a}_{t}=A(t) \tilde{a}_{t} d t=\lambda(t)^{\dagger} d B_{t}
$$

where $A(t)$ in the time-dependent $N \times N$ matrix with entries

$$
A_{j k}(t)=-\frac{1}{2} \lambda_{j}^{*}(t) \lambda_{k}(t)-i \omega_{k} \delta_{j k}
$$

The solution is

$$
\tilde{a}_{t}=\Phi(t) a-\Phi(t) \int_{0}^{t} \Phi(s)^{-1} \lambda(s)^{\dagger} d B_{s}
$$

which is given in terms of the transition matrix $\Phi(t)$ satisfying

$$
\frac{d}{d t} \Phi(t)=A(t) \Phi(t), \quad \Phi(0)=I_{N} .
$$

We then obtain the following vectorial generalization of (14)

$$
\begin{aligned}
\frac{d}{d t}\left\langle\Psi_{0}\left|\widetilde{U}(t)^{*}\left(I_{M} \otimes X(t)\right) \widetilde{U}(t)\right| \Psi_{0}\right\rangle \\
=\left\langle\Psi_{0}\left|\widetilde{U}(t)^{*} I_{M} \otimes\left(\mathcal{L}_{00}\left(X_{0}\right)+x_{00}+L^{*} x_{10}+x_{01} L+L^{*} x_{11} L\right) \widetilde{U}(t)\right| \Psi_{0}\right\rangle \\
\quad+\left\langle\Psi_{0}\left|a^{*} \Phi(t)^{\dagger} \lambda(t)^{*} \widetilde{U}(t)^{*} I_{M} \otimes\left(\mathcal{L}_{10}\left(X_{0}\right)+S^{*} x_{10}+S^{*} x_{11} L\right) \widetilde{U}(t)\right| \Psi_{0}\right\rangle \\
\quad+\left\langle\Psi_{0}\left|\widetilde{U}(t)^{*} I_{M} \otimes\left(\mathcal{L}_{01}\left(X_{0}\right)+x_{01} S+L^{*} x_{11} S\right) \widetilde{U}(t) \lambda(t) \Phi(t) a\right| \Psi_{0}\right\rangle \\
\quad+\left\langle\Psi_{0}\left|a^{*} \Phi(t)^{\dagger} \lambda(t)^{*} \widetilde{U}(t)^{*} I_{M} \otimes\left(\mathcal{L}_{11}\left(X_{0}\right)+S^{*} x_{11} S\right) \widetilde{U}(t) \lambda(t) \Phi(t) a\right| \Psi_{0}\right\rangle .
\end{aligned}
$$


Evidently, to get a prescribed set of pulses $\xi(t)$, we need to choose $\lambda(t)$ and the $\omega_{k}(t)$ 's such that

$$
\xi(t)=\lambda(t) \Phi(t)
$$

In general this is a difficult problem to solve, but for weak pulses the Magnus expansion [23] may offer a way to construct approximations. The set of functions $\xi_{k}$ that may be constructed in this way is expected to be fully general given the freedom of choosing the $\lambda_{k}$ and $\omega_{k},[24]$.

We now prepare the modulator in the initial state

$$
\phi_{0}=|\mathbf{n}\rangle=\left|n_{1}, \ldots, n_{N}\right\rangle
$$

where we have $n_{k}$ quanta in the $k$ th cavity mode.

This time we consider the family of expectations

$$
\tilde{M}_{t}^{\mathbf{n}_{l}, \mathbf{n}_{r}}(X)=\left\langle\mathbf{n}_{l} \otimes \psi_{0} \otimes \operatorname{vac}\left|\widetilde{U}_{t}^{*}\left(I_{M} \otimes X(t)\right) \widetilde{U}_{t}\right| \mathbf{n}_{r} \otimes \psi_{0} \otimes \mathrm{vac}\right\rangle
$$

for occupation sequences $\mathbf{n}_{l}=\left(n_{k, l}\right)$ and $\mathbf{n}_{r}=\left(n_{k, r}\right)$.

$$
\begin{aligned}
& \frac{d}{d t} \tilde{M}_{t}^{\mathbf{n}_{l}, \mathbf{n}_{r}}(X) \\
& =\tilde{M}_{t}^{\mathbf{n}_{l}, \mathbf{n}_{r}}\left(\mathcal{L}_{00}\left(X_{0}\right)+x_{00}(t)+L^{*} x_{10}+x_{01} L+x_{11} L\right) \\
& \quad+\sum_{k=1}^{N} \sqrt{n_{k, l}} \xi_{k}(t)^{*} \widetilde{M}_{t}^{\mathbf{n}_{l}-\delta_{k}, \mathbf{n}_{r}}\left(\mathcal{L}_{10}\left(X_{0}\right)+S^{*} x_{10}(t)+S^{*} x_{11} L\right) \\
& \quad+\sum_{j=1}^{N} \sqrt{n_{j, r}} \xi_{j}(t) \tilde{M}_{t}^{\mathbf{n}_{l}, \mathbf{n}_{r}-\delta_{j}}\left(\mathcal{L}_{01}\left(X_{0}\right)+x_{01}(t) S+L^{*} x_{11} S\right) \\
& \quad+\sum_{k=1}^{N} \sum_{j=1}^{N} \sqrt{n_{k, l}} \xi_{k}(t)^{*} \sqrt{n_{j, r}} \xi_{j}(t) \tilde{M}_{t}^{\mathbf{n}_{l}-\delta_{k}, \mathbf{n}_{r}-\delta_{j}}\left(\mathcal{L}_{11}\left(X_{0}\right)+S^{*} x_{11}(t) S\right)
\end{aligned}
$$

where now $\boldsymbol{\delta}_{k}$ is the occupation sequence where $n_{k}=1$ and all other terms are zero. We add sequences together in the obvious way so that $\mathbf{n}-\mu \boldsymbol{\delta}_{k}$ equals $\mathbf{n}$ if $\mu=0$, and $\left(n_{1}, \ldots\right.$, $\left.n_{k}-1, \ldots, n_{N}\right)$ if $\mu=1$.

By similar arguments to before, we see that system of expectations

$$
M_{t}^{\mathbf{n}_{l}, \mathbf{n}_{r}}(X)=\left\langle\psi_{0} \otimes \Xi\left(\mathbf{n}_{l}\right)\left|U_{t}^{*} X(t) U_{t}\right| \psi_{0} \otimes \Xi\left(\mathbf{n}_{r}\right)\right\rangle
$$

generate the same system of as the $\widetilde{M}_{t}^{\mathbf{n}_{l}, \mathbf{n}_{r}}(X)$ and so may be equated. We have therefore established that

Theorem 3 The quantum open system $G \sim(S, L, H)$ driven by input in the nonclassical state $\Xi(\mathbf{n})=\widehat{\bigotimes}_{k=1}^{N} \xi_{k}^{\otimes n_{k}}$ is replicated by the $N$ mode modulator of the linear form $M \sim$ $\left(I_{M}, \sum_{k} \lambda_{k}(t) a, \sum_{k} \omega_{k}(t) a_{k}^{*} a_{k}\right)$ with the initial state $\phi_{0}=|\mathbf{n}\rangle$ for the modulator and with $\lambda_{k}(t)$ and $\omega_{k}(t)$ chosen so that (21) holds. 


\section{Superposition principles}

We now make a basic observation.

Principle of Superimposed models For a fixed modulator $M$ - that is, a quantum open system with definite $\left(I_{M}, L_{M}, H_{M}\right)$ - suppose that initial states $\left|\phi_{0}^{A}\right\rangle,\left|\phi_{0}^{B}\right\rangle, \ldots$ replicate $\left|\Xi^{A}\right\rangle,\left|\Xi^{B}\right\rangle, \ldots$ respectively and are compatible in so far as

$$
\begin{aligned}
& \left\langle\phi_{0}^{A} \otimes \psi_{0} \otimes \operatorname{vac}\left|\tilde{U}(t)^{*}\left(I_{M} \otimes X(t)\right) \tilde{U}(t)\right| \phi_{0}^{B} \otimes \psi_{0} \otimes \mathrm{vac}\right\rangle \\
& \quad=\left\langle\psi_{0} \otimes \Xi^{A}\left|U(t)^{*} X(t) U(t)\right| \psi_{0} \otimes \Xi^{B}\right\rangle
\end{aligned}
$$

for each pair $A, B$. Then if the modulator is prepared in a normalized superposition $\left|\phi_{0}\right\rangle=$ $c_{A}\left|\phi_{0}^{A}\right\rangle+c_{B}\left|\phi_{0}^{B}\right\rangle+\cdots$ replicates the nonclassical state $|\Xi\rangle=c_{A}\left|\Xi^{A}\right\rangle+c_{B}\left|\Xi^{B}\right\rangle+\cdots$.

This follows automatically from the bra-ket structure of the matrix elements.

\subsection{Replicating cat states}

We would like to generate a superposition of coherent states (cat states [7-9])

$$
\Psi=\sum_{k} \gamma_{k}\left|\beta_{k}\right\rangle,
$$

with $\sum_{k, l} \gamma_{k}^{*} \gamma_{l} e^{\left\langle\beta_{k}, \beta_{l}\right\rangle}=1$ for normalization. It is easy to see that pairs of coherent states are compatible in the sense of the superposition principle.

We know that the modulator (with fixed structure $L_{M}=\lambda(t) a, H_{M}=\omega(t) a^{*} a$ ) prepared in coherent state $\left|\alpha_{k}\right\rangle$ will replicate the open system with coherent state $\left|\beta_{k}\right\rangle$ for the

$$
\beta_{k}(t)=\xi(t) \alpha_{k}
$$

The principle of superposition therefore implies that the initial state $\sum_{k} \gamma_{k}\left|\beta_{k}\right\rangle$ for the modulator will then replicate the cat state $\Psi=\sum_{k} \gamma_{k}\left|\beta_{k}\right\rangle$. Note that the $\beta_{k}$ that may be generated this way must take the form (25). This is somewhat restrictive since we cannot obtain independent pulse, only pulse which differ by the scale factors $\alpha_{k}$. However this already gives a wide class of cat states for practical purposes.

\section{Conclusion}

We have shown that the concept of a modulating filter 'coloring' a quantum vacuum noise input process may be usefully extended to the quantum domain. The technique in principle offers a way to generate nonclassical states, however, in a real sense we have now transferred the problem to one of preparing the ancillary oscillators in the appropriate nonclassical state and engineering the specific time dependent couplings, both of which remain difficult problems, but likely to be simpler than directly trying to produce designed nonclassical field states themselves. However, the power of this result is in modeling systems driven by nonclassical states which may be difficult to analyze directly using quantum stochastic techniques. Instead one replaces the problem with a cascaded model of modulator and system, with the modulator processing vacuum noise and feeding it into the system. We have shown that certain modulator models replicate the original system 
(A) Nonclassical input state

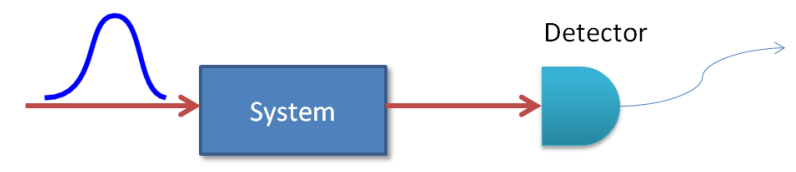

(B) Vacuum input state

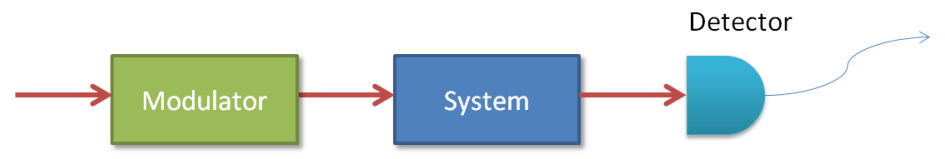

Figure 2 Continuous measurement of (A) the output of a system driven by nonclassical noise, and (B) and equivalent model from a modulated vacuum noise.

and noise model in a strong sense: that is we show that general quantum stochastic integral processes on the system plus noise space have identical averages in the original model with nonclassical input and in the modulated model with vacuum input. This in particular establishes equivalence of the system dynamics (effectively the same Ehrenfest equations for system observables) as well as equivalence of the outputs. The latter point is of major importance with regards to quantum trajectories (quantum filtering theory) since whenever we perform continuous measurements (e.g., quadrature homodyning, or photon counting), [25-30] on the output we have that the measurement processes of the original model and the modulate model are statistically identical. See Figure 2.

As a conceptual tool, this opens up the prospect of extending known results on quantum trajectories for vacuum inputs to models with nonclassical inputs. One such feature which we will address in a future publication is the issue of filter convergence, that is, when does the estimated conditional density operator converge to the true conditional density operator when one starts with the wrong initial state $\psi_{0}$ for the system - this has been treated for vacuum inputs [29], but is largely unknown in the case of nonclassical inputs.

Competing interests

The authors declare that they have no competing interests.

Authors' contributions

All authors contributed equally to the writing of this paper. All authors read and approved the final manuscript.

Author details

${ }^{1}$ Department of Physics, Aberystwyth University, Aberystwyth, Wales SY23 2BZ, UK. ${ }^{2}$ Department of Applied Mathematics, The Hong Kong Polytechnic University, Hong Kong, ACT, 0200, China.

\section{Acknowledgements}

The authors acknowledge support through the Royal Academy of Engineering UK and China scheme, and National Natural Science Foundation of China (NSFC) grant (Nos. 61374057) and a Hong Kong RGC grant (No. 531213).

Received: 14 January 2015 Accepted: 1 June 2015 Published online: 19 June 2015

References

1. Lvovsky Al, Hansen H, Aichele T, Benson O, Mlynek J, Schiller S. Phys Rev Lett. 2001;87:050402.

2. Pechal M, Huthmacher L, Eichler C, Zeytinoğlu S, Abdumalikov AA Jr, Berger S, Wallraff A, Filipp S. Phys Rev X. 2014;4:041010.

3. Flassig F, Kaniber M, Reithmaier G, Mueller K, Andrejew A, Gross R, Vuckovic J, Finley J. Proc. SPIE 9373, Quantum Dots and Nanostructures: Synthesis, Characterization, and Modeling XII. 2015;937305. 
4. Yuan Z, Kardynal BE, Stevenson RM, Shields AJ, Lobo CJ, Cooper K, Beattie NS, Ritchie DA, Pepper M. Science. 2002;295:102.

5. McKeever J, Boca A, Boozer AD, Miller R, Buck JR, Kuzmich A, Kimble HJ. Science. 2004;303:1992.

6. Eichler C, Bozyigit D, Lang C, Steffen L, Fink J, Wallraff A. Phys Rev Lett. 2011;106:220503.

7. Neergaard-Nielsen JS, Melholt Nielsen B, Hettich C, Mølmer K, Polzik ES. Phys Rev Lett. 2006;97:083604.

8. Ourjoumtsev A, Tualle-Brouri R, Laurat J, Grangier P. Science. 2006;312:83.

9. Ourjoumtsev A, Jeong H, Tualle-Brouri R, Grangier P. Nature. 2007:448:784.

10. Houck AA, Schuster I, Gambetta JM, Schreier JA, Johnson BR, Chow JM, Frunzio L, Majer J, Devoret MH, Girvin SM, Schoelkopf JM. Nature. 2006;449:328.

11. Ralph TC, Gilchrist A, Milburn GJ. Phys Rev A. 2003;68:042319.

12. Gisin N, Ribordy G, Tittel W, Zbinden H. Rev Mod Phys. 2002;74:145.

13. Anderson BDO, Moore JB. Optimal filtering. New York: Dover; 2005.

14. Carmichael HJ. An open systems approach to quantum optics. Lectures presented at the Universite Libre de Bruxelles, October 28 to November 4, 1991. Lecture notes in physics. vol. 18. 1994.

15. Gough JE, James MR, Nurdin HI, Combes J. Phys Rev A. 2012;86:043819.

16. Gough JE, James MR, Nurdin HI. New J Phys. 2014;16:075008.

17. Xue S, James MR, Shabani A, Ugrinovskii V, Petersen IR. Submitted to the 54th IEEE conference on decision and control. arXiv:1503.07999

18. Cirac Jl, Zoller P, Kimble HJ, Mabuchi H. Phys Rev Lett. 1997;78:3221.

19. Hudson RL, Parthasarathy KR. Commun Math Phys. 1984;93:301-23.

20. Evans MP, Hudson RL. In: Accardi L, von Waldenfels W, editors. Quantum Probability \& Applications, Volume III. Lecture Notes in Mathematics. vol. 1303. Heidelberg: Springer; 1988. p. 69-88.

21. Gough JE, James MR. Commun Math Phys. 2009;287:1109-32.

22. Gough JE, James MR. IEEE Trans Autom Control. 2009:54:2530,

23. Magnus W. Commun Pure Appl Math. 1954;7:649-73.

24. Wei J. J Math Phys. 1963:4:1337-41.

25. Belavkin VP. Radiotec Electron. 1980;25:1445-53.

26. Belavkin VP. J Multivar Anal. 1992:42:171-201.

27. Wiseman HM, Milburn GJ. Phys Rev Lett. 1993;70(5):548.

28. Wiseman HM, Milburn GJ. Phys Rev A. 1993;47(1):642.

29. van Handel R. Infin Dimens Anal Quantum Probab Relat Top. 2009;12:153-72.

30. Gough JE, Koestler C. Commun Stoch Anal. 2010;4(4):505-21.

\section{Submit your manuscript to a SpringerOpen ${ }^{\circ}$ journal and benefit from:}

- Convenient online submission

Rigorous peer review

- Immediate publication on acceptance

- Open access: articles freely available online

- High visibility within the field

- Retaining the copyright to your article 